\title{
Probabilistic seismic hazard analysis using logic tree approach- Patna district (India)
}

Anbazhagan P., Ketan Bajaj, Karanpreet Matharu, Sayed SR Moustafa, and Nassir S.N. Al-Arifi

Table S1 $M_{\max }$ corresponds to seismic sources used in the hazard analysis

\begin{tabular}{|c|c|c|c|c|c|}
\hline \multirow[b]{2}{*}{ Fault Name } & \multirow[b]{2}{*}{$M_{o b s}^{\max }$} & \multicolumn{3}{|c|}{$M_{\max }$ Estimation } & \multirow{2}{*}{$\begin{array}{c}M_{\max } \text { Taken for Hazard } \\
\text { Analysis }\end{array}$} \\
\hline & & $\begin{array}{c}\text { Kijko and } \\
\text { Sellevoll (1989) }\end{array}$ & $\begin{array}{c}\text { By Incremental } \\
\text { value }\end{array}$ & $\begin{array}{c}\text { By Regional Rupture } \\
\text { Characteristics }\end{array}$ & \\
\hline MBT & 8.00 & 8.0 & 8.50 & 8.1 & 8.2 \\
\hline MCT & 7.00 & 7.0 & 7.50 & 7.9 & 7.5 \\
\hline $\mathrm{s} 01$ & 5.40 & 5.4 & 5.90 & 6.9 & 6.2 \\
\hline $\mathrm{s} 02$ & 5.40 & 5.4 & 5.90 & 7.2 & 6.3 \\
\hline $\mathrm{s} 03$ & 5.20 & 5.2 & 5.70 & 7.1 & 6.1 \\
\hline s04 & 5.10 & 5.1 & 5.60 & 6.2 & 5.7 \\
\hline s05 & 5.20 & 5.2 & 5.70 & 6.9 & 6.1 \\
\hline s06 & 5.80 & 5.9 & 6.30 & 6.9 & 6.4 \\
\hline s07 & 5.80 & 6.6 & 6.30 & 6.3 & 6.4 \\
\hline $\mathrm{s} 08$ & 4.50 & 4.5 & 5.00 & 7.2 & 5.7 \\
\hline s09 & 5.60 & 5.6 & 6.10 & 6.9 & 6.3 \\
\hline $\mathrm{s} 10$ & 6.00 & 6.0 & 6.50 & 6.8 & 6.5 \\
\hline s100 & 4.60 & 4.9 & 5.10 & 5.0 & 5.0 \\
\hline s101 & 4.80 & 6.0 & 5.30 & 5.3 & 5.5 \\
\hline s102 & 5.50 & 6.1 & 6.00 & 5.7 & 5.9 \\
\hline s103 & 4.60 & 4.9 & 5.10 & 5.2 & 5.1 \\
\hline s104 & 5.40 & 5.5 & 5.90 & 6.7 & 6.1 \\
\hline s105 & 6.80 & 6.8 & 7.30 & 7.1 & 7.1 \\
\hline s106 & 6.80 & 6.8 & 7.30 & 7.2 & 7.1 \\
\hline s107 & 5.60 & 5.7 & 6.10 & 6.1 & 6.0 \\
\hline s108 & 4.50 & 4.7 & 5.00 & 5.5 & 5.1 \\
\hline s109 & 6.70 & 6.7 & 7.20 & 7.0 & 7.0 \\
\hline s11 & 5.60 & 5.6 & 6.10 & 7.1 & 6.4 \\
\hline s110 & 4.50 & 4.6 & 5.00 & 6.5 & 5.5 \\
\hline s111 & 6.00 & 7.0 & 6.50 & 6.3 & 6.6 \\
\hline s112 & 6.20 & 6.3 & 6.70 & 6.5 & 6.5 \\
\hline s113 & 4.50 & 6.2 & 5.00 & 6.0 & 5.8 \\
\hline s114 & 6.00 & 6.1 & 6.50 & 6.3 & 6.3 \\
\hline s115 & 5.20 & 5.4 & 5.70 & 6.0 & 5.7 \\
\hline s116 & 4.50 & 6.2 & 5.00 & 5.6 & 5.6 \\
\hline s117 & 4.80 & 4.9 & 5.30 & 5.0 & 5.1 \\
\hline s118 & 4.20 & 6.1 & 4.70 & 4.5 & 5.0 \\
\hline s119 & 4.50 & 4.7 & 5.00 & 6.1 & 5.4 \\
\hline s12 & 6.40 & 6.4 & 6.90 & 6.9 & 6.7 \\
\hline $\mathrm{s} 120$ & 5.50 & 5.7 & 6.00 & 6.4 & 6.1 \\
\hline s121 & 5.50 & 5.7 & 6.00 & 6.0 & 5.9 \\
\hline $\mathrm{s} 122$ & 4.90 & 5.1 & 5.40 & 5.3 & 5.3 \\
\hline s123 & 5.80 & 5.9 & 6.30 & 6.2 & 6.1 \\
\hline s124 & 5.60 & 5.8 & 6.10 & 6.0 & 6.0 \\
\hline s125 & 5.80 & 6.6 & 6.30 & 6.5 & 6.5 \\
\hline s126 & 5.60 & 5.8 & 6.10 & 5.9 & 5.9 \\
\hline s127 & 5.90 & 6.2 & 6.40 & 6.1 & 6.2 \\
\hline $\mathrm{s} 128$ & 6.40 & 6.4 & 6.90 & 7.3 & 6.9 \\
\hline s129 & 5.90 & 6.2 & 6.40 & 6.4 & 6.3 \\
\hline s13 & 5.20 & 5.2 & 5.70 & 6.9 & 6.0 \\
\hline s130 & 6.80 & 6.8 & 7.30 & 7.2 & 7.1 \\
\hline s131 & 5.30 & 5.4 & 5.80 & 6.6 & 6.0 \\
\hline s132 & 5.30 & 5.8 & 5.80 & 5.8 & 5.8 \\
\hline s133 & 4.70 & 4.6 & 5.20 & 5.1 & 5.0 \\
\hline s134 & 5.50 & 6.1 & 6.00 & 5.7 & 5.9 \\
\hline s135 & 4.20 & 6.1 & 4.70 & 4.5 & 5.0 \\
\hline
\end{tabular}




\begin{tabular}{|c|c|c|c|c|c|}
\hline $\mathrm{s} 136$ & 4.20 & 6.1 & 4.70 & 4.8 & 5.2 \\
\hline s137 & 4.30 & 4.4 & 4.80 & 4.7 & 4.6 \\
\hline $\mathrm{s} 138$ & 5.10 & 5.3 & 5.60 & 5.7 & 5.6 \\
\hline s139 & 5.60 & 5.6 & 6.10 & 6.9 & 6.3 \\
\hline s14 & 5.10 & 5.2 & 5.60 & 6.8 & 6.0 \\
\hline $\mathrm{s} 140$ & 5.30 & 5.5 & 5.80 & 5.8 & 5.7 \\
\hline s141 & 6.20 & 6.3 & 6.70 & 6.7 & 6.6 \\
\hline s142 & 5.00 & 6.4 & 5.50 & 5.4 & 5.7 \\
\hline s143 & 5.80 & 6.1 & 6.30 & 6.1 & 6.2 \\
\hline $\mathrm{s} 144$ & 4.50 & 6.2 & 5.00 & 5.0 & 5.4 \\
\hline $\mathrm{s} 145$ & 4.50 & 5.5 & 5.00 & 5.2 & 5.3 \\
\hline s146 & 5.30 & 5.3 & 5.80 & 6.9 & 6.1 \\
\hline s147 & 5.10 & 5.2 & 5.60 & 6.2 & 5.7 \\
\hline s148 & 5.80 & 5.9 & 6.30 & 6.5 & 6.3 \\
\hline s149 & 4.50 & 5.5 & 5.00 & 5.0 & 5.2 \\
\hline $\mathrm{s} 15$ & 5.50 & 5.6 & 6.00 & 7.0 & 6.3 \\
\hline $\mathrm{s} 150$ & 5.30 & 5.5 & 5.80 & 5.8 & 5.7 \\
\hline $\mathrm{s} 151$ & 5.10 & 5.6 & 5.60 & 5.6 & 5.6 \\
\hline $\mathrm{s} 152$ & 4.30 & 4.4 & 4.80 & 4.7 & 4.6 \\
\hline $\mathrm{s} 153$ & 4.30 & 4.4 & 4.80 & 4.7 & 4.6 \\
\hline $\mathrm{s} 154$ & 5.10 & 5.3 & 5.60 & 5.5 & 5.5 \\
\hline $\mathrm{s} 155$ & 4.50 & 5.5 & 5.00 & 5.0 & 5.1 \\
\hline $\mathrm{s} 156$ & 4.70 & 5.1 & 5.20 & 5.1 & 5.1 \\
\hline $\mathrm{s} 157$ & 5.80 & 5.9 & 6.30 & 6.8 & 6.4 \\
\hline $\mathrm{s} 158$ & 5.50 & 5.7 & 6.00 & 6.6 & 6.2 \\
\hline $\mathrm{s} 159$ & 4.50 & 6.2 & 5.00 & 6.0 & 5.7 \\
\hline $\mathrm{s} 16$ & 5.50 & 5.6 & 6.00 & 6.8 & 6.2 \\
\hline s160 & 5.00 & 5.2 & 5.50 & 6.0 & 5.6 \\
\hline s161 & 6.20 & 6.2 & 6.70 & 7.2 & 6.8 \\
\hline s162 & 4.50 & 4.6 & 5.00 & 6.4 & 5.4 \\
\hline s163 & 4.50 & 6.2 & 5.00 & 6.4 & 5.9 \\
\hline s164 & 4.50 & 4.6 & 5.00 & 6.1 & 5.3 \\
\hline s165 & 4.50 & 6.2 & 5.00 & 5.9 & 5.7 \\
\hline s166 & 4.50 & 4.7 & 5.00 & 5.5 & 5.1 \\
\hline s167 & 4.70 & 5.1 & 5.20 & 5.1 & 5.1 \\
\hline s168 & 4.80 & 6.0 & 5.30 & 5.1 & 5.4 \\
\hline s169 & 4.60 & 4.9 & 5.10 & 5.1 & 5.0 \\
\hline $\mathrm{s} 17$ & 5.10 & 5.2 & 5.60 & 6.8 & 6.0 \\
\hline $\mathrm{s} 170$ & 4.50 & 5.5 & 5.00 & 4.8 & 5.1 \\
\hline s171 & 5.10 & 5.2 & 5.60 & 6.2 & 5.7 \\
\hline $\mathrm{s} 172$ & 5.20 & 5.2 & 5.70 & 6.8 & 6.0 \\
\hline s173 & 4.50 & 6.2 & 5.00 & 5.2 & 5.4 \\
\hline s174 & 5.20 & 5.7 & 5.70 & 5.6 & 5.7 \\
\hline s175 & 4.20 & 6.1 & 4.70 & 4.5 & 5.0 \\
\hline $\mathrm{s} 176$ & 4.00 & 5.4 & 4.50 & 4.6 & 4.8 \\
\hline $\mathrm{s} 18$ & 5.50 & 6.1 & 6.00 & 6.8 & 6.4 \\
\hline $\mathrm{s} 19$ & 5.50 & 5.5 & 6.00 & 7.1 & 6.3 \\
\hline $\mathrm{s} 20$ & 5.50 & 5.5 & 6.00 & 6.9 & 6.2 \\
\hline s21 & 5.20 & 5.2 & 5.70 & 6.4 & 5.8 \\
\hline s22 & 5.20 & 5.2 & 5.70 & 6.3 & 5.8 \\
\hline s23 & 4.20 & 6.1 & 4.70 & 4.7 & 5.1 \\
\hline s24 & 4.50 & 6.2 & 5.00 & 5.7 & 5.6 \\
\hline s25 & 4.50 & 4.5 & 5.00 & 7.4 & 5.8 \\
\hline s26 & 5.40 & 5.5 & 5.90 & 6.2 & 5.9 \\
\hline s27 & 5.40 & 5.6 & 5.90 & 6.6 & 6.1 \\
\hline s28 & 5.20 & 5.2 & 5.70 & 6.8 & 6.0 \\
\hline s29 & 5.20 & 5.3 & 5.70 & 6.5 & 5.9 \\
\hline $\mathrm{s} 30$ & 5.40 & 6.0 & 5.90 & 6.5 & 6.2 \\
\hline s31 & 5.20 & 5.3 & 5.70 & 6.6 & 6.0 \\
\hline s32 & 5.20 & 5.7 & 5.70 & 6.6 & 6.1 \\
\hline s33 & 5.90 & 6.2 & 6.40 & 6.6 & 6.4 \\
\hline s34 & 5.40 & 5.4 & 5.90 & 7.2 & 6.3 \\
\hline $\mathrm{s} 35$ & 4.50 & 4.5 & 5.00 & 6.8 & 5.6 \\
\hline s36 & 5.50 & 5.5 & 6.00 & 6.9 & 6.2 \\
\hline s37 & 5.20 & 5.4 & 5.70 & 6.2 & 5.8 \\
\hline s38 & 4.70 & 5.1 & 5.20 & 6.3 & 5.6 \\
\hline
\end{tabular}




\begin{tabular}{|c|c|c|c|c|c|}
\hline s39 & 5.20 & 5.3 & 5.70 & 6.6 & 5.9 \\
\hline $\mathrm{s} 40$ & 5.20 & 5.3 & 5.70 & 6.1 & 5.7 \\
\hline $\mathrm{s} 41$ & 5.70 & 6.4 & 6.20 & 6.2 & 6.3 \\
\hline $\mathrm{s} 42$ & 5.70 & 6.0 & 6.20 & 6.2 & 6.1 \\
\hline $\mathrm{s} 43$ & 4.60 & 4.7 & 5.10 & 6.6 & 5.6 \\
\hline $\mathrm{s} 44$ & 5.30 & 5.3 & 5.80 & 7.1 & 6.2 \\
\hline $\mathrm{s} 45$ & 6.80 & 6.8 & 7.30 & 7.3 & 7.1 \\
\hline s46 & 5.10 & 5.3 & 5.60 & 6.1 & 5.7 \\
\hline $\mathrm{s} 47$ & 4.80 & 8.0 & 5.30 & 5.8 & 6.3 \\
\hline $\mathrm{s} 48$ & 5.30 & 5.4 & 5.80 & 6.6 & 6.0 \\
\hline $\mathrm{s} 49$ & 5.70 & 5.8 & 6.20 & 6.7 & 6.3 \\
\hline $\mathrm{s} 50$ & 6.10 & 6.1 & 6.60 & 6.8 & 6.5 \\
\hline s51 & 4.70 & 5.1 & 5.20 & 6.0 & 5.5 \\
\hline s52 & 5.90 & 6.0 & 6.40 & 6.6 & 6.3 \\
\hline s53 & 5.90 & 6.0 & 6.40 & 6.7 & 6.4 \\
\hline s54 & 6.20 & 6.2 & 6.70 & 6.9 & 6.6 \\
\hline s55 & 4.60 & 4.9 & 5.10 & 5.7 & 5.3 \\
\hline s56 & 5.70 & 5.8 & 6.20 & 6.1 & 6.0 \\
\hline s57 & 6.70 & 6.7 & 7.20 & 7.3 & 7.1 \\
\hline s58 & 5.40 & 5.4 & 5.90 & 7.2 & 6.3 \\
\hline s59 & 6.80 & 6.8 & 7.30 & 7.1 & 7.1 \\
\hline s60 & 5.40 & 5.4 & 5.90 & 7.2 & 6.3 \\
\hline s61 & 5.70 & 5.7 & 6.20 & 6.2 & 6.1 \\
\hline s62 & 7.00 & 7.0 & 7.50 & 7.3 & 7.3 \\
\hline s63 & 6.30 & 6.5 & 6.80 & 6.6 & 6.6 \\
\hline s64 & 4.90 & 5.0 & 5.40 & 6.6 & 5.7 \\
\hline s65 & 5.70 & 6.0 & 6.20 & 6.0 & 6.0 \\
\hline s66 & 5.00 & 5.5 & 5.50 & 5.4 & 5.4 \\
\hline s67 & 5.10 & 5.2 & 5.60 & 6.1 & 5.7 \\
\hline s68 & 5.00 & 5.2 & 5.50 & 5.7 & 5.5 \\
\hline s69 & 6.20 & 6.3 & 6.70 & 6.8 & 6.6 \\
\hline s70 & 4.90 & 5.4 & 5.40 & 5.4 & 5.4 \\
\hline s71 & 4.70 & 4.8 & 5.20 & 6.3 & 5.5 \\
\hline s72 & 4.80 & 4.9 & 5.30 & 5.9 & 5.4 \\
\hline s73 & 6.30 & 6.5 & 6.80 & 6.7 & 6.7 \\
\hline s74 & 5.60 & 6.3 & 6.10 & 6.1 & 6.2 \\
\hline s75 & 5.00 & 5.5 & 5.50 & 6.0 & 5.7 \\
\hline s76 & 6.10 & 6.1 & 6.60 & 6.5 & 6.4 \\
\hline s77 & 4.90 & 5.1 & 5.40 & 6.6 & 5.8 \\
\hline s78 & 6.10 & 7.2 & 6.60 & 6.3 & 6.7 \\
\hline s79 & 5.90 & 6.0 & 6.40 & 6.5 & 6.3 \\
\hline $\mathrm{s} 80$ & 4.50 & 6.2 & 5.00 & 5.0 & 5.4 \\
\hline $\mathrm{s} 81$ & 4.60 & 5.7 & 5.10 & 5.2 & 5.3 \\
\hline s82 & 4.90 & 5.0 & 5.40 & 6.6 & 5.7 \\
\hline s83 & 5.80 & 5.8 & 6.30 & 7.1 & 6.5 \\
\hline s84 & 4.80 & 5.2 & 5.30 & 5.4 & 5.3 \\
\hline $\mathrm{s} 85$ & 5.50 & 5.6 & 6.00 & 5.8 & 5.8 \\
\hline s86 & 4.80 & 5.2 & 5.30 & 5.3 & 5.3 \\
\hline s87 & 4.90 & 5.4 & 5.40 & 5.2 & 5.3 \\
\hline $\mathrm{s} 88$ & 4.60 & 4.9 & 5.10 & 5.3 & 5.1 \\
\hline s89 & 5.10 & 5.6 & 5.60 & 5.6 & 5.6 \\
\hline s90 & 5.80 & 6.6 & 6.30 & 6.4 & 6.4 \\
\hline s91 & 4.50 & 5.5 & 5.00 & 4.8 & 5.1 \\
\hline s92 & 4.30 & 4.4 & 4.80 & 4.7 & 4.6 \\
\hline s93 & 4.50 & 5.5 & 5.00 & 4.7 & 5.1 \\
\hline s94 & 4.80 & 5.2 & 5.30 & 5.2 & 5.3 \\
\hline s95 & 4.30 & 4.4 & 4.80 & 4.8 & 4.7 \\
\hline s96 & 4.70 & 4.6 & 5.20 & 5.1 & 5.0 \\
\hline s97 & 5.10 & 5.2 & 5.60 & 5.5 & 5.4 \\
\hline s98 & 4.50 & 5.5 & 5.00 & 4.9 & 5.1 \\
\hline s99 & 4.50 & 4.7 & 5.00 & 5.1 & 4.9 \\
\hline
\end{tabular}

